\title{
Qualitätstransparenz für Ärzte und Patienten
}

Die FMH engagiert sich für eine sinnvolle Transparenz in der Qualität ärztlichen Handelns. Sie setzt sich für die Veröffentlichung von aussagekräftigen, korrekt erhobenen und verständlich kommunizierten medizinischen Qualitätsdaten ein.

- Qualitätsmessung ist die Voraussetzung für Qualitätstransparenz. Damit Qualitätsindikatoren tatsächlich medizinische Behandlungsqualität repräsentieren, müssen sie gemeinsam mit der Ärzteschaft definiert werden.

- Qualitätstransparenz als Basis des internen Qualitätsmanagements führt zu Qualitätssteigerung in der medizinischen Behandlung und somit zu verbesserter Patientenversorgung und Patientensicherheit.

- Die Veröffentlichung von Qualitätsdaten soll der Vergleichbarkeit ärztlicher Leistungserbringung dienen. Auf diese Weise kann einem reinen Preiswettbewerb ein Qualitätswettbewerb zur Seite gestellt werden.

\section{Die Ausgangslage}

In Spitälern, Praxen und Ärztenetzwerken wird eine Vielzahl von Qualitätsaktivitäten realisiert. Diese sind oftmals für die Patienten und die interessierte Öffentlichkeit zu wenig sichtbar. Die FMH sieht hier Handlungsbedarf und setzt sich für mehr Qualitätstransparenz in der medizinischen Leistungserbringung ein. Einen wichtigen Teil der medizinischen Qualitätsarbeit erbringen die Ärztinnen und Ärzte. Deshalb müssen sie mitbestimmen, welche Qualitätsdaten erhoben, ausgewertet und veröffentlicht werden. Innerhalb der Ärzteschaft hält die FMH eine koordinierende Funktion inne. Sie steht in Austausch mit den Fachgesellschaften, welche die fachspezifischen Qualitätsaktivitäten und die Indikatoren zur Qualitätsmessung definieren. Die FMH vertritt die Ärzteschaft und erstellt in Zusammenarbeit mit den Tarifpartnern die Grundlage für eine sinnvolle Qualitätstransparenz.

\section{Die Position der FMH}

\section{Qualitätstransparenz dient dem internen} Qualitätsmanagement

Qualitätstransparenz ist die Basis für das interne Qualitätsmanagement. Eine systematische und kontinuierliche Qualitätsarbeit trägt dazu bei, eigene Stärken und Schwächen zu erkennen. Für eine wirksame, zweckmässige und wirtschaftliche medizinische Leistungserbringung braucht es sinnvolle Qualitätsmessungen, die Aussagen zur Behandlungsqualität ermöglichen. Primäres Ziel muss der Nutzenzuwachs und nicht die Kostensenkung sein. Ein wichtiger Nutzen der Quali-

Korrespondenz: Generalsekretariat FMH Abteilung DDQ Elfenstrasse 8 CH-3000 Bern 15 ddq@fmh.ch

\section{Qualitätswettbewerb statt reiner Preiswettbewerb} Die Messung und Veröffentlichung von Qualitätsindikatoren dient der Vergleichbarkeit der Leistungserbringer. Dem Patienten soll auf diese Weise die Auswahl des passenden Arztes bzw. der passenden Institution erleichtert werden. Die FMH setzt sich für einen unverfälschten Wettbewerb ein: Qualitätsdaten müssen korrekt erhoben und ausgewertet werden. Bereiche der medizinischen Behandlung, die nicht im Fokus der Qualitätsmessung liegen, dürfen nicht vernachlässigt werden. Qualitätsaktivitäten sollen nicht nur kurzfristige Ziele verfolgen. Innovationen müssen weiterhin gefördert werden. Es ist ein Anliegen der FMH, gemeinsam mit dem Gesetzgeber und den Tarifpartnern Voraussetzungen zu schaffen, die einen fairen Qualitätswettbewerb in der medizinischen Leistungserbringung ermöglichen.

\section{Qualitätstransparenz für die Patienten}

Die Veröffentlichung von Qualitätsdaten darf nicht zum Ziel haben, massenmedialen Ansprüchen zu genügen. Vielmehr gilt es, ernsthaft auf die Bedürfnisse von Patientinnen und Patienten einzugehen. Die FMH unterstützt eine zielgruppenspezifische Transparenz. Nicht alle Qualitätsdaten sind für die verschiedenen Anspruchsgruppen gleich interpretierbar und nützlich. Es eignen sich somit nicht alle Qualitätsdaten für die breite Veröffentlichung. Die FMH hilft mit gezielten Studien Erkenntnisse zum Informationsbedürfnis der Patientinnen und Patienten im Bereich medizinischer Behandlungsqualität zu gewinnen.

\section{Qualität auch bei der Datenerhebung, -auswertung und -publikation}

Inkorrekte oder inkorrekt analysierte medizinische Qualitätsdaten verhindern, dass die Ärzteschaft die gute Qualität ihrer Leistungen aufzeigen kann. Sie führen nicht zur gewünschten Verbesserung der Qualität und verunmöglichen einen gesunden Wettbewerb unter den Leistungserbringern. Nicht zuletzt werden die Patienten getäuscht und ihr Vertrauen in das Gesundheitssystem wird geschwächt. Eine positive Wirkung kann nur die Veröffentlichung von korrekten und für die Empfänger verständlichen Qualitätsdaten erzielen. Zahlen werden erst aussagekräftig, wenn sie von adäquaten Interpretationshilfen begleitet werden. Bei der Publikation von Qualitätsdaten sollen deshalb die hierfür von einer Arbeitsgruppe unter der Leitung der Schweizer Akademie für medizinische Wissenschaften erarbeiteten Kriterien zur Anwendung kommen. Die Veröffentlichung von Qualitätsdaten ist insbesondere nur dann sinnvoll, wenn diese tatsächlich Behandlungsqualität repräsentieren. Damit Indikatoren Aussagen über die Qualität ärztlichen Handelns zulassen, müssen sie deshalb zwingend zusammen mit der Ärzteschaft definiert werden. 